\title{
Clinical and therapeutic relevance of PIM1 kinase in gastric cancer
}

\author{
Benedict Yan - Ee Xuan Yau $\cdot$ Sanjay Samanta $\cdot$ Chee Wee Ong $\cdot$ Kol Jia Yong $\cdot$ Lai Kuan Ng • \\ Bhaskar Bhattacharya $\cdot$ Kiat Hon Lim · Richie Soong • Khay Guan Yeoh • Niantao Deng • \\ Patrick Tan $\cdot$ Yulin Lam $\cdot$ Manuel Salto-Tellez $\cdot$ Singapore Gastric Cancer Consortium
}

Received: 22 November 2010/Accepted: 11 September 2011/Published online: 13 October 2011

(C) The International Gastric Cancer Association and The Japanese Gastric Cancer Association 2011

\begin{abstract}
Background Gastric cancer is a leading cause of cancerrelated mortality, and chemotherapeutic options are currently limited. PIM1 kinase, an oncogene that promotes tumorigenesis in several cancer types, might represent a novel therapeutic target in gastric cancer.

Methods We studied the expression and genomic status of PIM1 in human primary gastric normal and tumor tissue
\end{abstract}

Electronic supplementary material The online version of this article (doi:10.1007/s10120-011-0097-2) contains supplementary material, which is available to authorized users.

B. Yan $(\bowtie) \cdot$ L. K. Ng · M. Salto-Tellez $(\bowtie)$

Department of Pathology, National University Health System and National University of Singapore, 5 Lower Kent Ridge

Road, Singapore 119074, Singapore

e-mail: tranceblues@gmail.com

M. Salto-Tellez

e-mail: patmst@nus.edu.sg

E. X. Yau - C. W. Ong - K. J. Yong · B. Bhattacharya .

R. Soong $\cdot$ M. Salto-Tellez

Cancer Science Institute, National University of Singapore,

Singapore, Singapore

S. Samanta $\cdot$ Y. Lam

Department of Chemistry, National University of Singapore,

3 Science Drive 3, Singapore 117543, Singapore

K. H. Lim

Department of Pathology, Singapore General Hospital,

Singapore, Singapore

K. G. Yeoh

Department of Medicine, National University Health System and

National University of Singapore, Singapore, Singapore

N. Deng · P. Tan

Duke-NUS Graduate Medical School, Singapore, Singapore samples by immunohistochemistry and array-based comparative genomic hybridization (aCGH). To ascertain whether PIM1 expression predicted susceptibility to PIM1 kinase-specific inhibition, the cytotoxic effect of a previously reported PIM1-specific small molecular inhibitor (K00135) was investigated in two gastric cancer cell lines with high (IM95) and undetectable (NUGC-4) PIM1 expression levels.

Results PIM1 expression was exclusively nuclear in normal gastric epithelial cells, while aberrant expression/ localization (decreased nuclear and/or increased cytoplasmic expression) was observed in $75.6 \%$ (68/90) of the human gastric cancer tissue samples, with a significant inverse correlation between nuclear and cytoplasmic expression levels. Clinicopathological analyses revealed that decreased nuclear PIM1 expression correlated with poorer survival and greater depth of tumor invasion, while increased cytoplasmic PIM1 expression correlated inversely with the presence of lymphovascular invasion. Highlevel PIM1 amplification was identified in $10.5 \%$ of gastric cancers by aCGH. K00135 impaired the survival of IM95, while it had no significant effect on NUGC-4 survival.

Conclusion Our findings demonstrate the clinical and therapeutic relevance of PIM1 in gastric cancers, and suggest that PIM1 represents a potential therapeutic target.

Keywords PIM1 - Gastric cancer · Targeted therapy · Amplification

\section{Introduction}

Gastric cancer is a major cause of cancer-related morbidity and mortality, being the second leading cause of cancerrelated deaths globally [1]. A significant number of patients 
present with advanced disease, leading to poor survival outcomes [2]. To date, the number of effective molecular therapeutics available against gastric cancer is limited [3]. There is a need to identify potential novel therapeutic targets in gastric cancer.

PIM1 is a serine/threonine kinase [4] that was initially discovered to play an oncogenic role in T-cell lymphomagenesis in mice [5]. The oncogenetic mechanisms of PIM1 include the promotion of cell cycle progression [6, 7], the suppression of apoptosis [8], and the enhancement of drug resistance [9]. Overexpression of PIM1 also results in genomic instability and polyploidy [10, 11]. PIM1 has been implicated in tumorigenesis in many cancer types, including myeloid leukemias [12], B-cell lymphomas [13, 14], and prostate [15] and head and neck cancers [16], and there has been growing interest in developing small molecular inhibitors of PIM1 as potential therapeutic agents [17-20].

PIM1 expression has been reported to correlate with prognosis in gastric cancer [21, 22]; however, its potential as a candidate therapeutic target has not been explored in detail. Of note, the PIM1 gene is located on chromosome 6 p21 [23], a region that is amplified in some gastric cancers [24]. In this study, our objectives were to study the immunohistochemical expression of PIM1 in gastric cancer tissue samples, identify whether genomic PIM1 amplification was present in primary gastric cancer tissue samples and cell lines, and determine whether PIM1 is a candidate therapeutic target by performing drug testing in cell lines using a previously reported potent and specific small molecular inhibitor of PIM1, K00135 [12].

\section{Materials and methods}

\section{Clinical samples}

\section{National University Hospital (NUH)}

Ninety surgically resected specimens of primary human matched gastric tumor and normal samples were selected from the archives of the Department of Pathology, National University Hospital, Singapore. Tissue microarray construction, immunohistochemistry and clinicopathological analyses were performed on this cohort. Twenty-four matched biopsies with intestinal metaplasia were analyzed by immunohistochemistry on complete sections. Patient consent for research, and ethics approval from the Institutional Review Board were obtained.

\section{National Cancer Centre of Singapore (NCCS)}

One hundred and five anonymized primary human gastric tumors were obtained from the NCCS tissue repository, with approval from the local Ethics Committee and signed patient informed consent. Array-based comparative genomic hybridization $(\mathrm{aCGH})$ was performed on this cohort. Tumor content in all samples was confirmed to be $>50 \%$ by frozen sections. Genomic DNAs from pooled blood lymphocytes obtained from healthy female and male subjects were used as normalization controls.

Tissue microarray construction and immunohistochemistry

Tissue cylinders with a diameter of $1 \mathrm{~mm}$ were punched from formalin-fixed, paraffin-embedded samples of tumor and normal areas of the donor tissue blocks and deposited into a recipient block using a tissue-arraying instrument (Beecher Instruments ${ }^{\circledR}$, Silver Spring, MD, USA). Paraffin tissue microarray sections of $4 \mu \mathrm{m}$ were deparaffinized and hydrated in graded ethanols. Heat-induced epitope retrieval of tissues was performed by boiling at $120^{\circ} \mathrm{C}$ in $\mathrm{pH} 6$ citrate buffer target retrieval solution (Dako, Glostrup, Denmark) for $5 \mathrm{~min}$ in a pressure cooker. Non-specific signal was blocked by peroxidase block (Dako) for $10 \mathrm{~min}$ at room temperature, followed by protein block (Dako) for $30 \mathrm{~min}$ at room temperature. Anti-PIM1 primary monoclonal antibody (Epitomics, Burlingame, CA, USA) was incubated at room temperature for $1 \mathrm{~h}$ in a humidified chamber, followed by horseradish peroxidase (HRP)-conjugated secondary antibody (Dako) incubation at room temperature for $30 \mathrm{~min}$. Antibody binding was revealed by diaminobenzidine (DAB) (Dako). Tissue sections were counterstained with hematoxylin, dehydrated in graded ethanols, and mounted.

For the immunohistochemical analysis, the intensity of PIM1 expression in gastric cancers was scored relative to that in normal gastric epithelial cells. Therefore, nuclear PIM1 expression was scored as being either strong or decreased, while cytoplasmic PIM1 expression was scored as being either increased or weak/undetectable.

\section{Clinicopathological data and analysis}

Clinicopathological data comprising distant metastasis, lymphovascular invasion, perineural invasion, lymph nodal metastasis, tumor size, depth of invasion, and survival were available for 76 cases from the NUH cohort. Differences between these clinical parameters and PIM1 immunohistochemical expression were assessed using the Fisher's exact test and, whenever appropriate, the $\chi^{2}$ test. Their correlations were examined using Spearman's rank coefficient test. Odds ratio was estimated by means of crosstabulation analysis. Survival curves were plotted using the Kaplan-Meier method and compared using the log-rank test. A Cox proportional hazards model for the hazard ratio 
was used for the evaluation of survival in a multivariate analysis. All statistical analyses were performed using an SPSS package (version 15.0 for Windows, SPSS Inc., Chicago, IL, USA) with significance set at the 5\% level.

Array-CGH assays and copy number analysis

One hundred and five primary gastric tumors were profiled using Agilent Human Genome 244A CGH Microarrays (Agilent Technologies, Santa Clara, CA, USA) containing approximately 240,000 distinct 60-mer oligonucleotide probes spanning the entire human genome. Sample labeling and hybridizations were performed according to the manufacturer's instructions. Hybridized slides were scanned on an Agilent DNA Microarray Scanner (Agilent Technologies) and images were extracted using Agilent Feature Extraction software. Copy number variation levels for the PIM1 locus were analyzed using Nexus software (http:// www.biodiscovery.com/index/nexus).

\section{Cell culture}

Two gastric cancer cell lines, IM95 and NUGC-4, were maintained in advanced Dulbecco's modified Eagle medium (DMEM) (Invitrogen Life Technologies, Carlsbad, CA, USA) supplemented with $2 \%$ Glutamax (Invitrogen) and heat-inactivated fetal bovine serum (Invitrogen), and Advanced RPMI 1640 (Invitrogen) supplemented with 1\% Glutamax and $2 \%$ heat-inactivated fetal bovine serum, respectively. A prostate cancer cell line, PC-3 (ATCC, Manassas, VA, USA), was cultured in F-12 nutrient mixture (HAM) (Invitrogen) supplemented with 10\% heatinactivated fetal bovine serum under a humidified atmosphere of $5 \% \mathrm{CO}_{2}$ at $37^{\circ} \mathrm{C}$.

Quantitative real-time polymerase chain reaction (PCR)

Total RNAs of IM95, NUGC-4, and PC-3 were isolated with an RNeasy Mini Kit (Qiagen, Hilden, Germany), according to the manufacturer's instructions and reverse transcribed using High Capacity cDNA Reverse Transcription (RT) kit with RNase inhibitor. Quantitative real-time PCR (Q-PCR) was performed with an ABI Prism 7900HT thermocycler (Applied Biosystems, Foster City, CA, USA), using PIMI and GAPDH FAM dye-labeled TaqMan ${ }^{\circledR}$ probes based on TaqMan ${ }^{\circledR}$ fluorescence methodology. All the reactions were conducted in duplicate. All reagents used for RT and Q-PCR were purchased from Applied Biosystems. PIM1 mRNA was normalized to GAPDH mRNA.

\section{Western blotting}

Cells were lysed with protease inhibitor in NP-40 lysis buffer, briefly sonicated on ice, and subjected to high-speed centrifugation for $1 \mathrm{~min}$. Cell lysates were resolved by $12 \%$ sodium dodecyl sulfate-polyacrylamide gel electrophoresis (SDS-PAGE) and transferred to a nitrocellulose membrane. The membranes were blocked in 5\% skimmed milk in phosphate-buffered saline $0.1 \%$ Tween-20 (PBS-T) and proteins were probed with anti-PIM1 (Epitomics) and $\beta$-actin (Santa Cruz Biotechnology, Santa Cruz, CA, USA) primary antibodies, followed by HRP-conjugated antirabbit or anti-mouse secondary antibody. The probed proteins were detected with Luminol (Santa Cruz) on exposure to X-ray film.

Fluorescence in situ hybridization (FISH)

Cells were harvested and fixed with Carnoy's fixative (3:1 methanol:acetic acid) on coated slides. The cells were digested with protease solution $(4 \mathrm{mg} / \mathrm{ml}$ protease in $0.2 \mathrm{~N}$ HCl) (Vysis, Abbott Molecular, Des Plaines, IL, USA) for $15 \mathrm{~min}$ at $37^{\circ} \mathrm{C}$. A PIM1 probe (BAC clone RP11-200O9; Enzo Life Sciences, Farmingdale, NY, USA) and a CEP6 probe (Vysis, Abbott Molecular) were added. Denaturation and hybridization were performed using ThermoBrite (Abbott Molecular, Des Plaines, IL, USA). Following overnight hybridization at $37^{\circ} \mathrm{C}$, the slides were washed in $50 \%$ formamide $/ 2 \times$ saline-sodium citrate $(\mathrm{SSC})$ at $37^{\circ} \mathrm{C}$ for $20 \mathrm{~min}$ and $1 \times \mathrm{SSC}$ at room temperature for $15 \mathrm{~min}$
Scheme 1 Synthesis of K00135

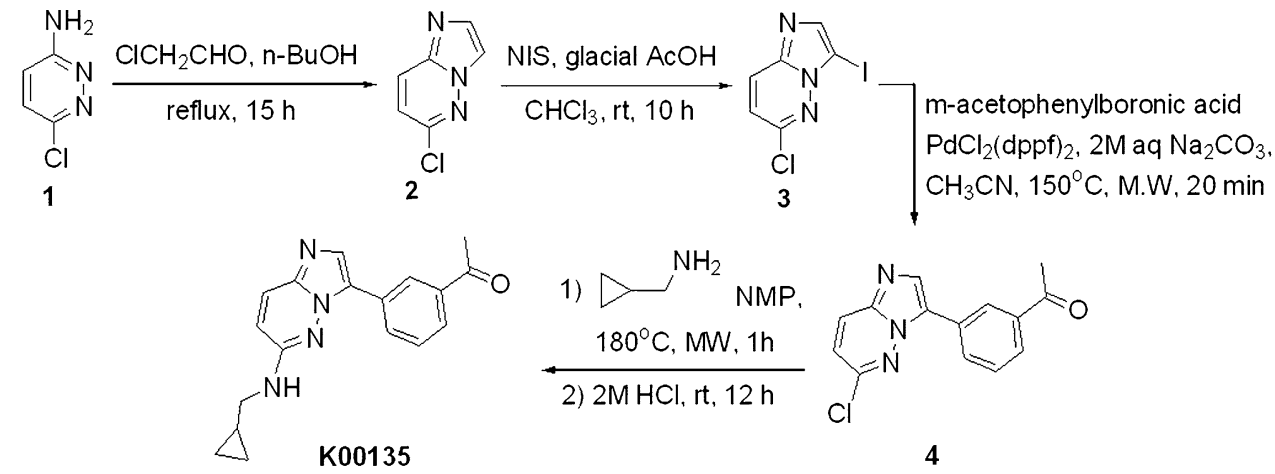


and counterstained with $125 \mathrm{ng} / \mathrm{ml} \mathrm{4,6-diamidino-2-phe-}$ nylindole (DAPI) (Vysis, Abbott Molecular). The slides were analyzed using an Olympus BX61 microscope (Olympus, Tokyo, Japan) equipped with DAPI, Spectrum Orange, Spectrum Green, and double-band pass filters for simultaneous visualization of the Spectrum Green and Spectrum Orange signals. Images were captured using CytoVision version 3.93 software (Applied Imaging, Newcastle upon Tyne, UK). One hundred cells were scored and the ratio of PIMI to CEP6 signals was calculated. Gene amplification was defined by a mean PIM1/CEP6 copy number of $>2.2$ or by a mean PIMI copy number of $>6$ per cell according to previously reported criteria [25].

\section{K00135 synthesis}

Briefly, 1-(3-(6-(cyclopropylmethylamino)imidazo [1,2-b]pyridazin-3-yl)phenyl)ethanone (K00135) was synthesized as described in Scheme 1. Treatment of 3-amino6-chloropyridazine $\mathbf{1}$ with chloroacetaldehyde gave intermediate 6-chloroimidazo[1,2-b]pyridazine $\mathbf{2}$, which was subsequently selectively iodinated at the 2-position with $N$-iodosuccinimide (NIS) to yield the key intermediate 3. Suzuki coupling of $\mathbf{3}$ with $m$-acetophenylboronic acid under microwave irradiation occurred selectively at the 2-iodo position to provide intermediate $\mathbf{4}$. Reaction of $\mathbf{4}$ with cyclopropylmethylamine under microwave conditions followed by treatment with dilute $\mathrm{HCl}$ afforded the target compound K00135. A detailed description of the synthetic process can be found in the Supplementary Information.

\section{Treatment with K00135}

Cells in $90 \mu \mathrm{l}$ medium were seeded (3,000 cells/well) onto 96-well microtiter plates (Nunc, Rochester, NY, USA). After $24 \mathrm{~h}, 10 \mu \mathrm{l}$ of medium containing K00135 with graded concentrations ranging from 10 to $10,000 \mathrm{nM}$ was added to the wells. The effect on cell numbers was assessed using the (3-(4,5-dimethylthiazol-2-yl)-5-(3-carboxymethoxyphenyl)-2-(4-sulfophenyl)-2H-tetrazolium, inner salt) (MTS) CellTiter $96^{\circledR}$ (Promega, Madison, WI, USA) assay at $72 \mathrm{~h}$ post-treatment. The $\mathrm{IC}_{50}$ was calculated as the drug
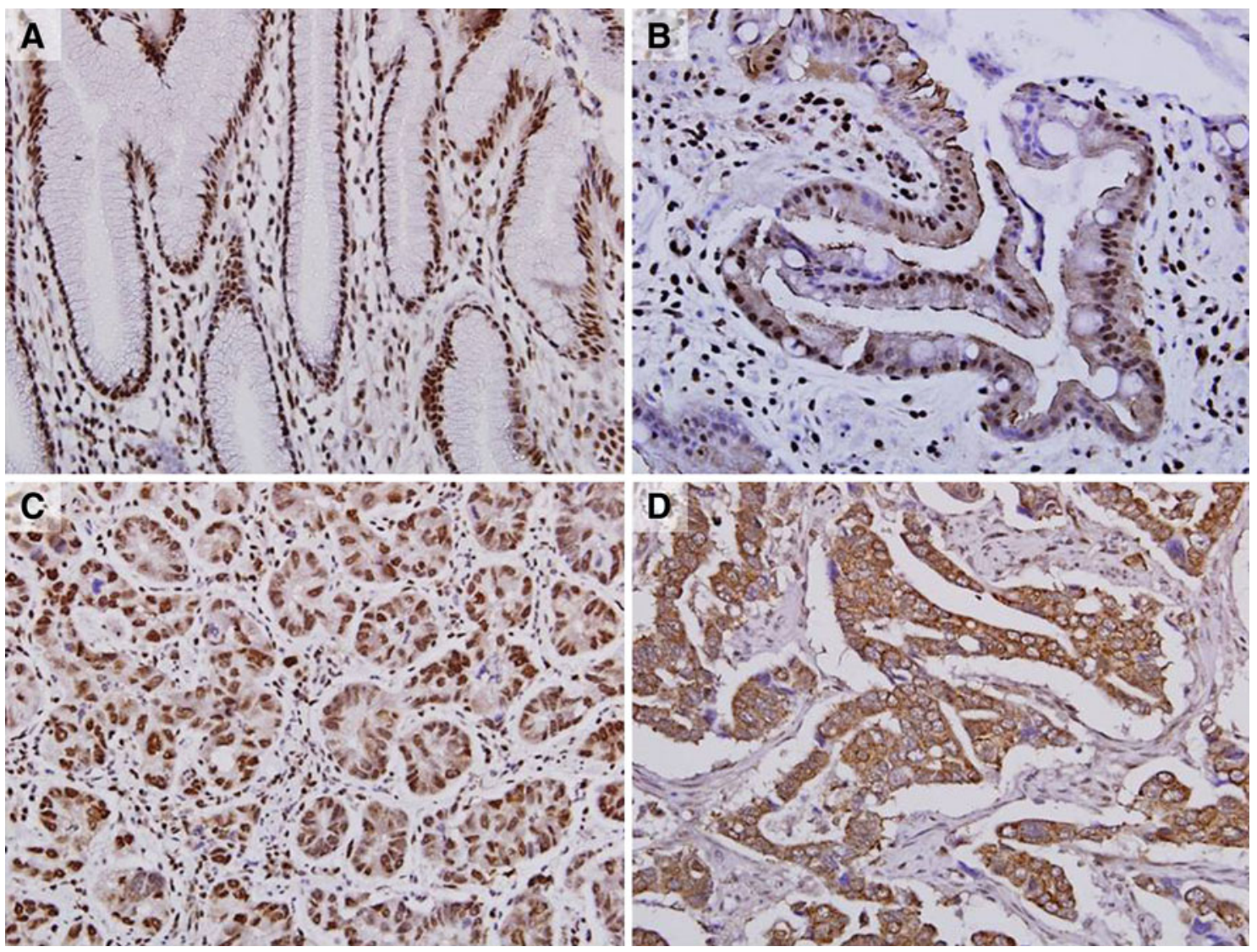

Fig. 1 PIM1 immunohistochemistry in normal and tumor gastric samples showing a strong nuclear staining in normal gastric epithelial cells, b nuclear and cytoplasmic staining in intestinal metaplasia, c strong nuclear staining in tumor cells, d strong cytoplasmic staining in tumor cells (original magnification $\mathrm{x} 400$ ) 
concentration that inhibited cell proliferation by $50 \%$ compared with vehicle controls.

\section{Results}

Aberrant PIM1 expression/localization in primary gastric cancers and clinicopathological correlation

In normal gastric epithelial cells, PIM1 expression was strongly nuclear with undetectable or very weak cytoplasmic staining (Fig. 1a). Nuclear PIM1 expression was present in all cases of intestinal metaplasia, with $45.8 \%$ (11/24) showing concomitant cytoplasmic staining (Fig. 1b). In primary gastric cancer samples from the NUH cohort, we observed aberrant PIM1 expression/localization, manifested as either decreased nuclear expression or increased cytoplasmic expression (Fig. 1c, d), in $75.6 \%$ (68/90) of gastric cancers (Table 1). There was a significant inverse correlation between nuclear and cytoplasmic PIM1 expression levels $(p=0.033)$.

Complete clinicopathological information was available for 76 cases from the NUH cohort. Table 2 illustrates the demographic and clinicopathological characteristics of the NUH cohort. When analyzed against clinicopathological parameters, decreased nuclear PIM1 expression correlated with poorer survival $(p=0.036)$ (Fig. 2a) and greater depth of tumor invasion $(p=0.003)$. Multivariate analysis demonstrated a significant correlation between decreased nuclear PIM1 expression and poorer survival $(p=0.025)$. Increased cytoplasmic PIM1 expression correlated inversely with the presence of lymphovascular invasion $(p=0.047)$. No correlation between cytoplasmic PIM1 expression and survival was identified (Fig. 2b). Tables 3 , 4 , and 5 provide detailed analyses of nuclear and cytoplasmic PIM1 expression against the various clinicopathological parameters.

Table 1 PIM1 nuclear and cytoplasmic expression in gastric cancer samples

\begin{tabular}{llll}
\hline & $\begin{array}{l}\text { Strong } \\
\text { nuclear } \\
\text { expression }\end{array}$ & $\begin{array}{l}\text { Decreased } \\
\text { nuclear } \\
\text { expression }\end{array}$ & Total \\
\hline $\begin{array}{l}\text { Increased cytoplasmic expression } \\
\begin{array}{l}\text { Weak/undetectable cytoplasmic } \\
\text { expression }\end{array}\end{array}$ & 9 & 31 & 40 \\
Total & 31 & 28 & 50 \\
\hline
\end{tabular}

Focal high-level PIM1 genomic amplification in primary gastric cancers

As gains of chromosome 6p21 have been reported in approximately $10 \%$ of gastric cancers previously [24], we were interested in investigating whether PIMI genomic amplification was present in our primary tumors. By aCGH analysis, PIMI was found to be amplified in $10.5 \%$ $(11 / 105)$ and $24.8 \%(26 / 105)$ of the primary gastric cancers, using a threshold of high and low stringency, respectively (Fig. 3).

K00135 cytotoxicity correlates with PIM1 mRNA and protein expression levels in gastric cancer cell lines

The endogenous levels of PIM1 mRNA and protein were measured by Q-PCR and immunoblot assays. The IM95 and NUGC-4 gastric cancer cell lines displayed high and low/ undetectable endogenous PIM1 expression, respectively, both at the mRNA (Fig. 4a) and protein levels (Fig. 4b). As

Table 2 Demographic and clinicopathological characteristics of National University Hospital (NUH) cohort

\begin{tabular}{lc}
\hline Parameters & $n(\%)$ \\
\hline Age (years) & \\
$<65$ & $30(39.5)$ \\
$\geq 65$ & $46(60.5)$ \\
Gender & \\
Male & $56(73.4)$ \\
Female & $20(26.3)$ \\
Stage & \\
I and II & $38(50.0)$ \\
III and IV & $38(50.0)$ \\
Distant metastasis & \\
Absent & $68(89.5)$ \\
Present & $8(10.5)$ \\
Lymphovascular invasion & \\
Absent & $32(42.1)$ \\
Present & $44(33.9)$ \\
Perineural invasion & \\
Absent & $50(65.8)$ \\
Present & $26(34.2)$ \\
Lymph nodal metastasis & \\
Absent & $22(28.9)$ \\
Present & $54(71.1)$ \\
Tumor size & \\
$<5$ cm & $36(47.4)$ \\
Depth of invasion (pT) & $40(52.6)$ \\
1 and 2 & \\
3 and 4 & \\
\hline & \\
\hline & \\
\hline & \\
\hline
\end{tabular}


A

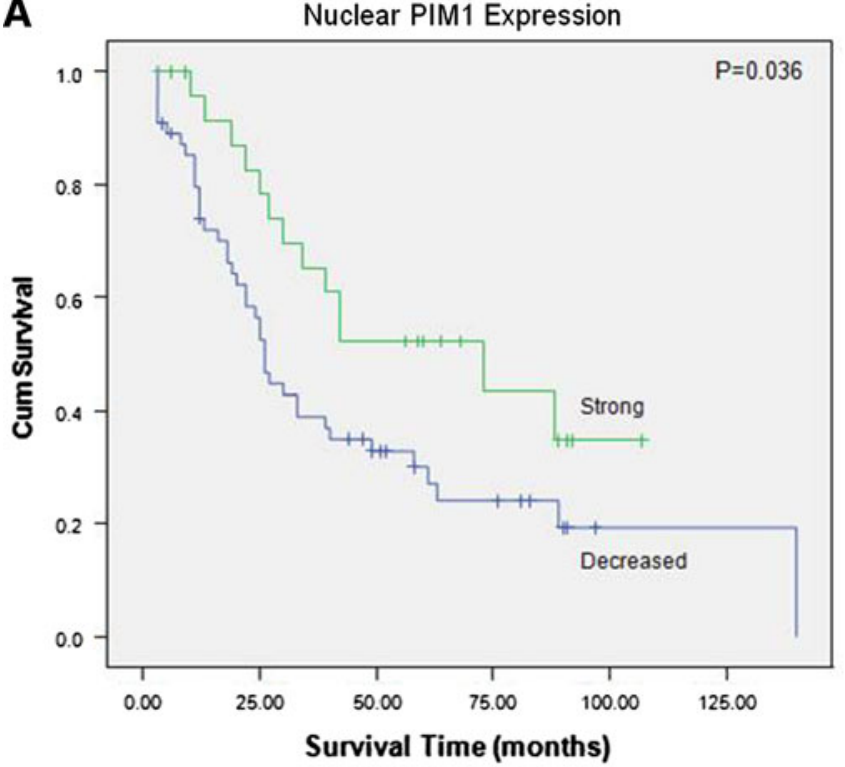

B

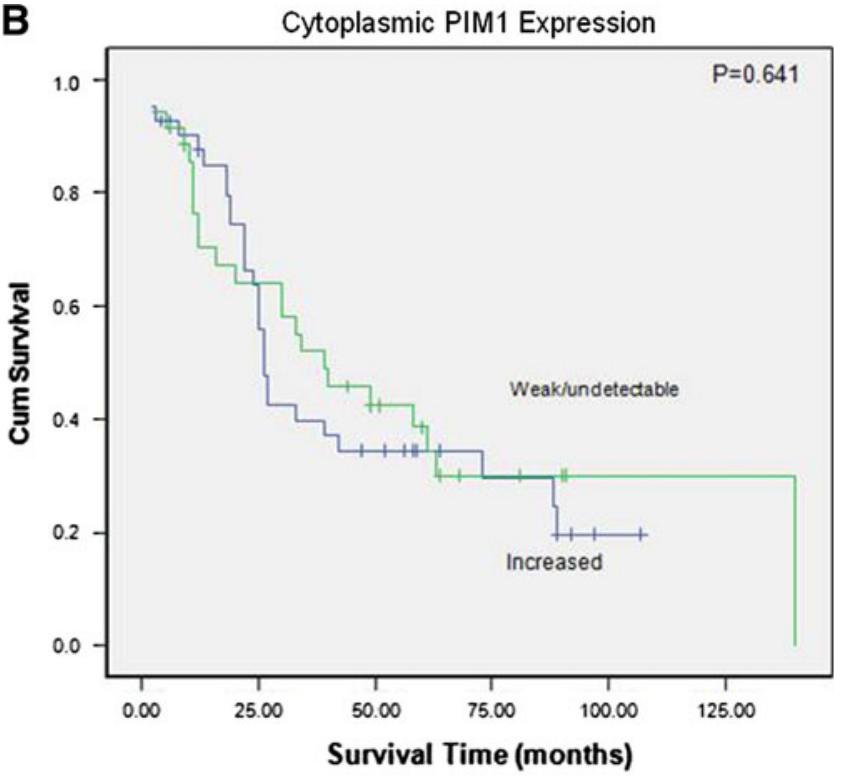

Fig. 2 Kaplan-Meier survival curve showing the relationship of a nuclear and b cytoplasmic PIM1 expression with survival. Cum cumulative

Table 3 Clinicopathological parameters in relation to nuclear PIM1 expression

\begin{tabular}{lccc}
\hline Parameters & $\begin{array}{l}\text { Decreased nuclear } \\
\text { PIM1 expression }\end{array}$ & $\begin{array}{l}\text { Strong nuclear } \\
\text { PIM1 expression }\end{array}$ & $p$ value \\
\hline Stage & & & \\
I and II & 25 & 13 & 0.805 \\
III and IV & 27 & 11 & \\
Distant metastasis & & 0.702 \\
Absent & 47 & 21 & \\
Present & 5 & 3 & 0.141 \\
Lymphovascular invasion & & \\
Absent & 27 & 17 & 0.437 \\
Present & 25 & 7 & \\
Perineural invasion & & \\
Absent & 36 & 14 & 0.787 \\
Present & 16 & 10 & \\
Lymph nodal metastasis & & \\
Absent & 16 & 6 & \\
Present & 36 & 18 & \\
Tumor size & & 13 & \\
$<5$ cm & 23 & 20 & \\
$\geq 5$ cm & 29 & & \\
Depth of invasion (pT) & & \\
1 and 2 & 24 & & \\
3 and 4 & 28 & & \\
\hline
\end{tabular}

Value in bold is significant

PIM1 gene amplification might have accounted for the differential expression, FISH analysis was performed to ascertain this. By FISH analysis, most cells in both cell lines showed a similar number of PIMI and CEP6 signals (2-4
Table 4 Clinicopathological parameters in relation to cytoplasmic PIM1 expression

\begin{tabular}{|c|c|c|c|}
\hline Parameters & $\begin{array}{l}\text { Weak/undetectable } \\
\text { cytoplasmic PIM1 } \\
\text { expression }\end{array}$ & $\begin{array}{l}\text { Increased } \\
\text { cytoplasmic PIM1 } \\
\text { expression }\end{array}$ & $p$ value \\
\hline \multicolumn{4}{|l|}{ Stage } \\
\hline I and II & 19 & 19 & \multirow[t]{2}{*}{0.490} \\
\hline III and IV & 22 & 16 & \\
\hline \multicolumn{4}{|c|}{ Distant metastasis } \\
\hline Absent & 36 & 32 & \multirow[t]{2}{*}{0.719} \\
\hline Present & 5 & 3 & \\
\hline \multicolumn{4}{|c|}{ Lymphovascular invasion } \\
\hline Absent & 13 & 19 & \multirow[t]{2}{*}{0.047} \\
\hline Present & 28 & 16 & \\
\hline \multicolumn{4}{|c|}{ Perineural invasion } \\
\hline Absent & 28 & 22 & \multirow[t]{2}{*}{0.619} \\
\hline Present & 13 & 13 & \\
\hline \multicolumn{4}{|c|}{ Lymph nodal metastasis } \\
\hline Absent & 12 & 10 & \multirow[t]{2}{*}{0.947} \\
\hline Present & 29 & 25 & \\
\hline \multicolumn{4}{|l|}{ Tumor size } \\
\hline$<5 \mathrm{~cm}$ & 19 & 17 & \multirow[t]{2}{*}{0.846} \\
\hline$\geq 5 \mathrm{~cm}$ & 22 & 18 & \\
\hline \multicolumn{4}{|c|}{ Depth of invasion (pT) } \\
\hline 1 and 2 & 26 & 18 & \multirow[t]{2}{*}{0.291} \\
\hline 3 and 4 & 15 & 17 & \\
\hline
\end{tabular}

Value in bold is significant

signals) per cell (Fig. 4c), with colocalization of both PIMI and CEP6 signals. Occasionally in both cell lines, the PIMI signals did not colocalize with the CEP6 signals, suggesting 
translocation of the PIM1 gene to regions outside chromosome 6. No significant difference in the PIMI gene copy number was discerned between IM95 (PIMI/CEP6 ratio 1.09) and NUGC-4 (PIMI/CEP6 ratio 1.1). The mean PIM1 copy numbers/cell were 2.2 and 2.5 for IM95 and NUGC-4, respectively, excluding the presence of PIMI amplification in either cell line.

K00135 has previously been reported to be a potent and specific inhibitor of PIM1, with an $\mathrm{IC}_{50}$ of $0.12 \mu \mathrm{mol} / 1$ [12]. To investigate whether PIM1 affected cell growth, we exposed the gastric cancer cell lines to various

Table 5 Multivariate analysis for survival according to clinicopathological features and nuclear PIM1 expression

\begin{tabular}{llll}
\hline Parameter & HR & $95 \%$ CI & $p$ \\
\hline Age $\geq 65$ years & 1.76 & $0.916-3.365$ & 0.090 \\
Distant metastasis & 3.765 & $1.414-10.03$ & $\mathbf{0 . 0 0 8}$ \\
Lymphovascular invasion & 0.801 & $0.386-1.661$ & 0.551 \\
Perineural invasion & 0.562 & $0.266-1.187$ & 0.131 \\
Lymph nodal metastasis & 0.520 & $0.227-1.190$ & 0.121 \\
Tumor size $\geq 5$ cm & 1.45 & $0.710-2.962$ & 0.308 \\
Depth of invasion (pT3/4) & 1.418 & $0.730-2.753$ & 0.302 \\
Decreased nuclear PIM1 expression & 2.45 & $1.127-5.348$ & $\mathbf{0 . 0 2 5}$ \\
\hline
\end{tabular}

$H R$ hazard ratio, $C I$ confidence interval

Values in bold are significant concentrations of the drug and measured cell viability subsequently. K00135 impaired the survival of IM95 $\left(\mathrm{IC}_{50}=2.4 \mu \mathrm{mol} / \mathrm{l}\right)$ (Fig. $4 \mathrm{~d}$ ), while it had no effect on NUGC-4 gastric cancer cells ( IC $\left._{50}>10 \mu \mathrm{mol} / \mathrm{l}\right)$.

\section{Discussion}

Two studies have earlier pointed to a link between PIM1 and gastric carcinogenesis [21, 22], and our present study builds on their findings by providing a more detailed immunohistochemical, clinicopathological, genomic analysis with in vitro functional studies. In contrast to Warnecke-Eberz et al. [21], who reported undetectable to weak cytoplasmic PIM1 expression in normal gastric epithelial cells, we detected strong nuclear PIM1 expression in normal gastric epithelial cells. We feel that technical factors might account for the observed difference-a polyclonal antibody was employed in their study, while a monoclonal antibody (the specificity of which was confirmed on Western immunoblotting) was utilized in ours.

We observed aberrant expression/localization of PIM1 in a population of primary gastric cancer samples, manifested as either decreased nuclear or increased cytoplasmic expression. Decreased nuclear expression correlated with greater depth of tumor invasion and poorer survival, while

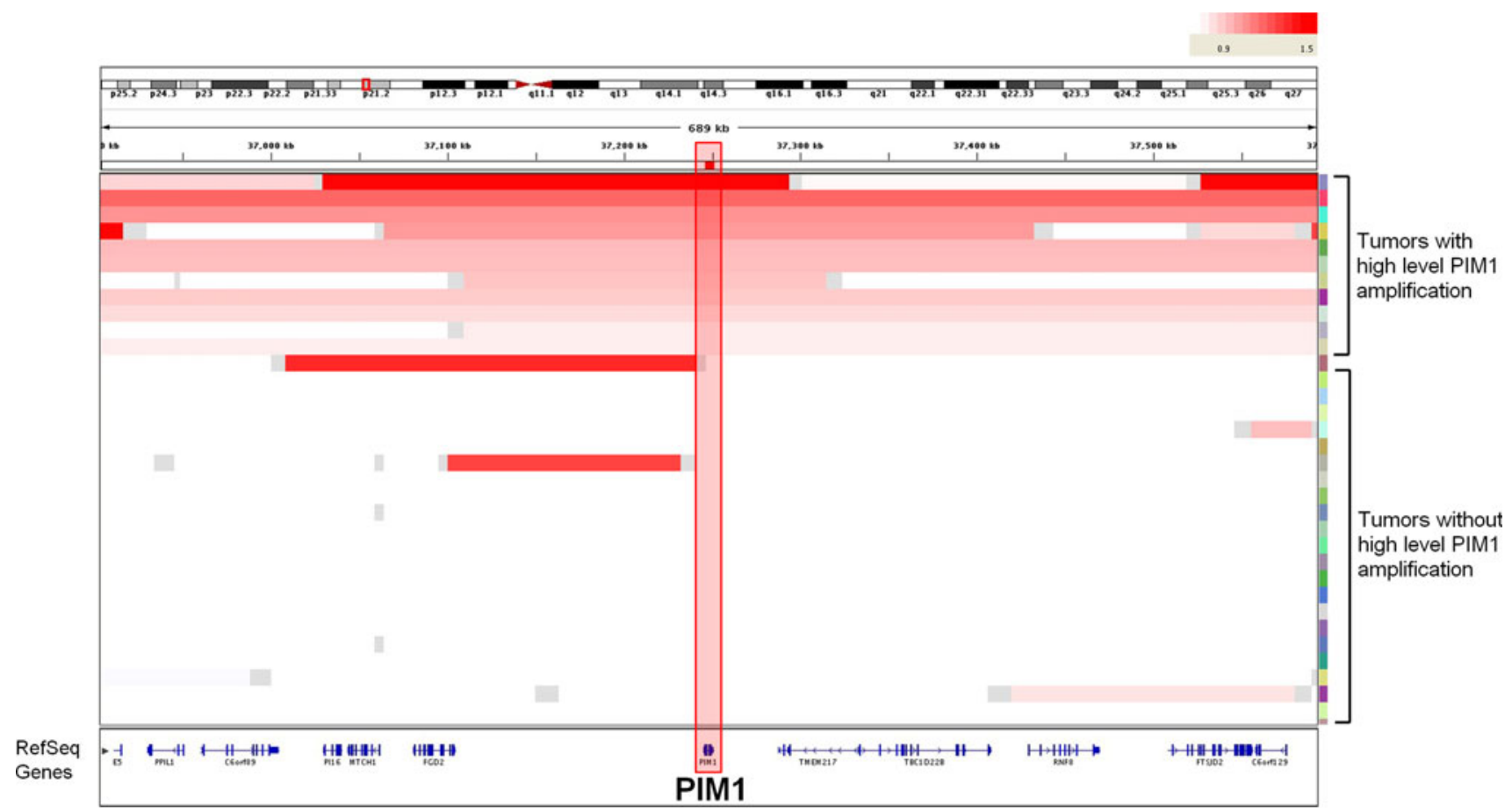

Fig. 3 Copy number profiles of PIM1 in 33 primary gastric tumors. Overlap of diverse amplification regions across multiple samples covers PIM1. Eleven tumors with high-level copy number amplification of PIMI are shown in red. The color gradient depicts the extent of copy number amplification. Genomic position and surrounding genes are indicated accordingly 


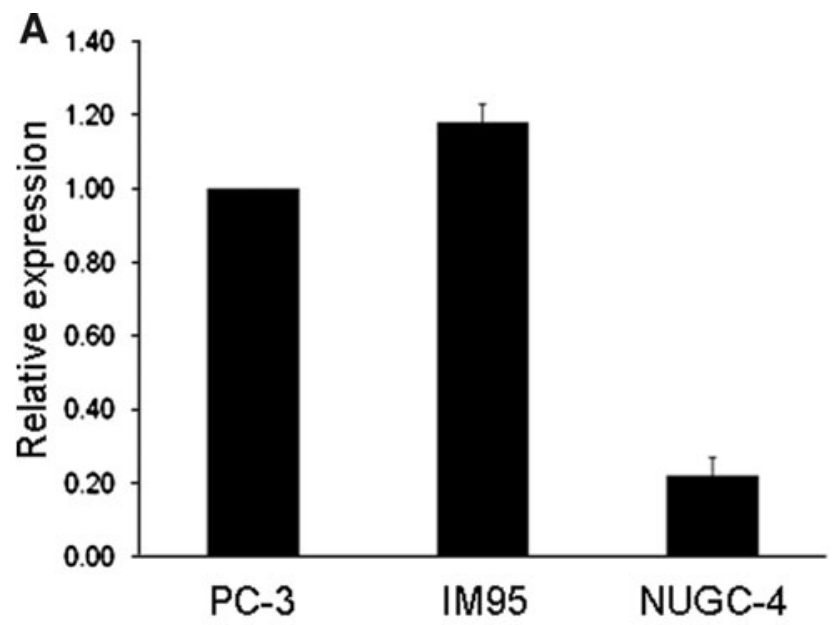

C
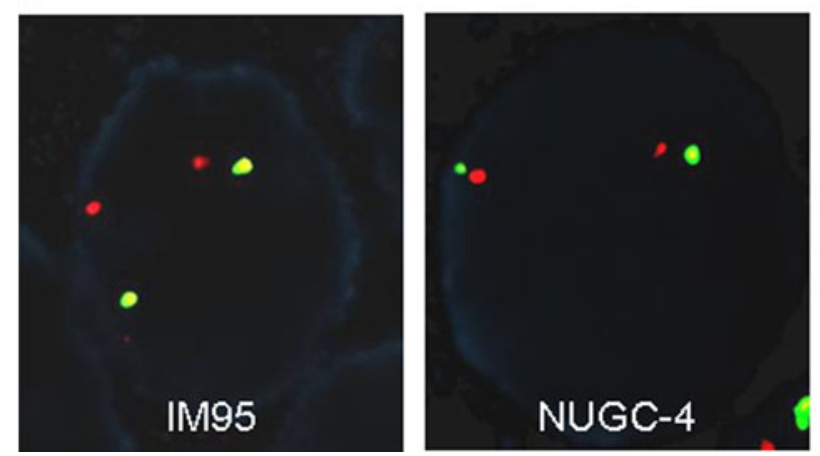

Fig. 4 Endogenous PIM1 a mRNA and $\mathbf{b}$ protein expression levels in IM95 and NUGC-4 cell lines. PC-3 was included as a positive control for PIM1 expression for both quantitative real-time polymerase chain reaction (Q-PCR) and Western blotting (WB). c PIM1 fluorescence in

increased cytoplasmic expression correlated inversely with the presence of lymphovascular invasion. In particular, multivariate analysis showed decreased nuclear PIM1 expression to be a strong independent prognostic factor. Increased cytoplasmic PIM1 expression was identified in some cases of intestinal metaplasia, a pre-malignant condition [26], suggesting that aberrant PIM1 expression occurs early during gastric carcinogenesis. Our findings raise the question as to the biological function of subcellular PIM1 localization, and its role in gastric tumorigenesis.

PIM1 is known to phosphorylate both nuclear and cytoplasmic target proteins. Exclusively nuclear target proteins include histone $\mathrm{H} 3$ [27], while exclusively cytoplasmic target proteins include Bad $[8,28]$ and BCRP/ ABCG2 [9]. Target proteins that may localize in either the nucleus or cytoplasm include Runx3 [29], p21 ${ }^{\text {Cip1/WAF1 }}$ [30], C-TAK1 [6, 31], p27 ${ }^{\text {Kip1 }}$ [7], and C-myc [32]. Although PIM1 has been shown to promote oncogenesis through the phosphorylation of nuclear target proteins such as histone $\mathrm{H} 3$, based on our observation that normal gastric epithelial cells display strong nuclear PIM1 expression, it is
B
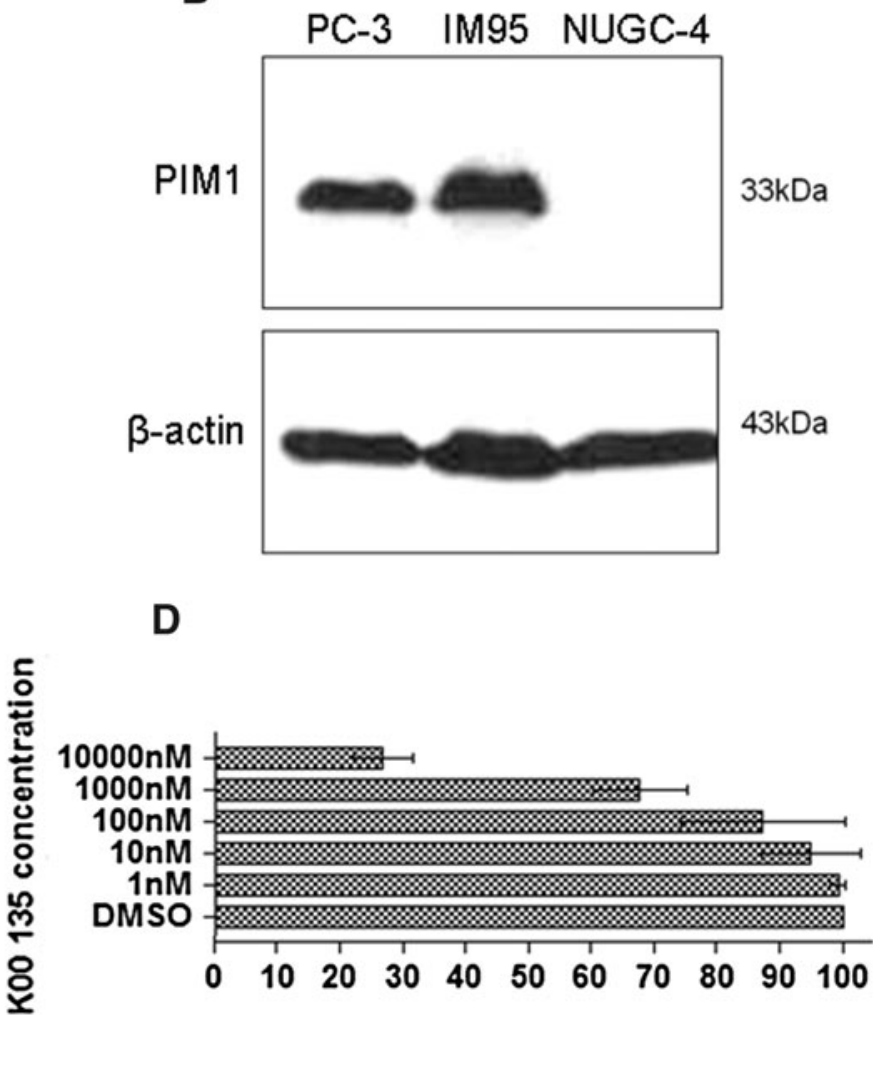

situ hybridization (FISH) in IM95 and NUGC-4. Orange and green signals represent PIM1 and CEP6 probes, respectively. d Effect of K00135 treatment on IM95

unlikely that nuclear PIM1 functions as an oncogene in gastric cancers. Loss of nuclear expression and gain of cytoplasmic expression appear to be the likely mechanisms of oncogenesis in gastric cancers, although further work will be needed to verify this hypothesis. Our finding that cytoplasmic PIM1 expression correlates inversely with the presence of lymphovascular invasion is potentially interesting in view of a recent report that implicates PIM1 in invasion and metastasis [33].

The identification of high-level PIMI genomic amplification by aCGH suggests that overexpression and subsequent gain of PIM1 function might occur in a subset of gastric cancers. It is interesting to note that PIMI genomic amplification has been previously reported in a subset of B-cell non-Hodgkin's lymphomas [14]. It will be important in future work to elucidate the functional significance of PIM1 genomic amplification, as this phenomenon suggests oncogenic dependency on PIM1 and therefore raises the possibility that PIM1 might be a potential molecular therapeutic target in a subset of gastric cancers. Such a paradigm is exemplified by HER2 in breast [34] and gastric cancers [35]. 
As an extension of our findings from the clinical samples, we therefore also sought to understand whether PIM1 expression had therapeutic significance in gastric cancers. Specifically, we wished to study whether the expression of PIM1 mediated cell survival in gastric cancers, and whether this expression predicted response to anti-PIM1-specific therapy, the latter objective being especially important because we also noted that $31.1 \%$ (28/90) of gastric cancers showed decreased nuclear and low/undetectable cytoplasmic expression which might predict decreased sensitivity or resistance to PIM1-specific treatment. Our in vitro work with gastric cancer cell lines showed that PIM1 expression mediated survival, and PIM1 expression levels correlated with susceptibility to PIM1-specific treatment. Together, these findings suggest that PIM1 expression in gastric cancers might have therapeutic relevance. The observation that the differential PIM1 mRNA and protein expression in IM95 and NUGC-4 gastric cancer cell lines was not due to a PIM1 gene copy number difference suggests that other mechanisms, such as epigenetic or post-translational regulation [36], might regulate PIM1 levels.

In summary, we report aberrant PIM1 expression/ localization and high-level PIM1 genomic amplification in a population of gastric cancer tissue samples, and that susceptibility to PIM1 inhibition following treatment with a PIM1-specific small molecular inhibitor correlates with PIM1 mRNA and protein expression in gastric cancer cell lines. Our findings demonstrate the clinical and therapeutic relevance of PIM1 in gastric cancers, warranting further study of PIM1 as a potential therapeutic target.

Acknowledgments MS-T received funding support from SCS grants MN-05, MN-05R, and MN-77, awarded by the Singapore Cancer Syndicate, Agency for Science, Technology and Research, Singapore; the Cancer Science Institute of Singapore; and the Singapore Gastric Cancer Consortium.

\section{References}

1. Parkin DM, Bray F, Ferlay J, Pisani P. Global cancer statistics, 2002. CA Cancer J Clin. 2005;55:74-108.

2. Fock KM, Talley N, Moayyedi P, Hunt R, Azuma T, Sugano K, et al. Asia-Pacific consensus guidelines on gastric cancer prevention. J Gastroenterol Hepatol. 2008;23:351-65.

3. Hede K. Gastric cancer: trastuzumab trial results spur search for other targets. J Natl Cancer Inst. 2009;101:1306-7.

4. Padma R, Nagarajan L. The human PIM-1 gene product is a protein serine kinase. Cancer Res. 1991;51:2486-9.

5. Cuypers HT, Selten G, Quint W, Zijlstra M, Maandag ER, Boelens W, et al. Murine leukemia virus-induced T-cell lymphomagenesis: integration of proviruses in a distinct chromosomal region. Cell. 1984;37:141-50.

6. Bachmann M, Kosan C, Xing PX, Montenarh M, Hoffmann I, Moroy T. The oncogenic serine/threonine kinase Pim-1 directly phosphorylates and activates the G2/M specific phosphatase Cdc25C. Int J Biochem Cell Biol. 2006;38:430-43.
7. Morishita D, Katayama R, Sekimizu K, Tsuruo T, Fujita N. Pim kinases promote cell cycle progression by phosphorylating and down-regulating p27Kip1 at the transcriptional and posttranscriptional levels. Cancer Res. 2008;68:5076-85.

8. Aho TL, Sandholm J, Peltola KJ, Mankonen HP, Lilly M, Koskinen PJ. Pim-1 kinase promotes inactivation of the pro-apoptotic Bad protein by phosphorylating it on the Ser112 gatekeeper site. FEBS Lett. 2004;571:43-9.

9. Xie Y, Xu K, Linn DE, Yang X, Guo Z, Shimelis H, et al. The 44-kDa Pim-1 kinase phosphorylates BCRP/ABCG2 and thereby promotes its multimerization and drug-resistant activity in human prostate cancer cells. J Biol Chem. 2008;283:3349-56.

10. Roh M, Franco OE, Hayward SW, van der Meer R, Abdulkadir SA. A role for polyploidy in the tumorigenicity of Pim-1expressing human prostate and mammary epithelial cells. PLoS ONE. 2008;3:e2572.

11. Roh M, Gary B, Song C, Said-Al-Naief N, Tousson A, Kraft A, et al. Overexpression of the oncogenic kinase Pim-1 leads to genomic instability. Cancer Res. 2003;63:8079-84.

12. Pogacic V, Bullock AN, Fedorov O, Filippakopoulos P, Gasser C, Biondi A, et al. Structural analysis identifies imidazo[1, 2-b]pyridazines as PIM kinase inhibitors with in vitro antileukemic activity. Cancer Res. 2007;67:6916-24.

13. Ionov Y, Le X, Tunquist BJ, Sweetenham J, Sachs T, Ryder J, et al. Pim-1 protein kinase is nuclear in Burkitt's lymphoma: nuclear localization is necessary for its biologic effects. Anticancer Res. 2003;23:167-78.

14. Sivertsen EA, Galteland E, Mu D, Holte H, Meza-Zepeda L, Myklebost $\mathrm{O}$, et al. Gain of chromosome $6 \mathrm{p}$ is an infrequent cause of increased PIM1 expression in B-cell non-Hodgkin's lymphomas. Leukemia. 2006;20:539-42.

15. Cibull TL, Jones TD, Li L, Eble JN, Ann Baldridge L, Malott SR, et al. Overexpression of Pim-1 during progression of prostatic adenocarcinoma. J Clin Pathol. 2006;59:285-8.

16. Beier UH, Weise JB, Laudien M, Sauerwein H, Gorogh $\mathrm{T}$. Overexpression of Pim-1 in head and neck squamous cell carcinomas. Int J Oncol. 2007;30:1381-7.

17. Holder S, Zemskova M, Zhang C, Tabrizizad M, Bremer R, Neidigh JW, et al. Characterization of a potent and selective small-molecule inhibitor of the PIM1 kinase. Mol Cancer Ther. 2007;6:163-72.

18. Cheney IW, Yan S, Appleby T, Walker H, Vo T, Yao N, et al. Identification and structure-activity relationships of substituted pyridones as inhibitors of Pim-1 kinase. Bioorg Med Chem Lett. 2007; 17:1679-83.

19. Pierce AC, Jacobs M, Stuver-Moody C. Docking study yields four novel inhibitors of the protooncogene Pim-1 kinase. J Med Chem. 2008;51:1972-5.

20. Tong Y, Stewart KD, Thomas S, Przytulinska M, Johnson EF, Klinghofer $\mathrm{V}$, et al. Isoxazolo[3,4-b]quinoline-3,4(1H, 9H)-diones as unique, potent and selective inhibitors for Pim-1 and Pim2 kinases: chemistry, biological activities, and molecular modeling. Bioorg Med Chem Lett. 2008;18:5206-8.

21. Warnecke-Eberz U, Bollschweiler E, Drebber U, Metzger R, Baldus SE, Holscher AH, et al. Prognostic impact of protein overexpression of the proto-oncogene PIM-1 in gastric cancer. Anticancer Res. 2009;29:4451-5.

22. Chen CN, Lin JJ, Chen JJ, Lee PH, Yang CY, Kuo ML, et al. Gene expression profile predicts patient survival of gastric cancer after surgical resection. J Clin Oncol. 2005;23:7286-95.

23. Nagarajan L, Louie E, Tsujimoto Y, ar-Rushdi A, Huebner K, Croce CM. Localization of the human pim oncogene (PIM) to a region of chromosome 6 involved in translocations in acute leukemias. Proc Natl Acad Sci USA. 1986;83:2556-60.

24. Gorringe KL, Boussioutas A, Bowtell DD. Novel regions of chromosomal amplification at $6 \mathrm{p} 21,5 \mathrm{p} 13$, and $12 \mathrm{q} 14$ in gastric 
cancer identified by array comparative genomic hybridization. Genes Chromosomes Cancer. 2005;42:247-59.

25. Okamoto W, Okamoto I, Yoshida T, Okamoto K, Takezawa K, Hatashita E, et al. Identification of $\mathrm{c}-\mathrm{Src}$ as a potential therapeutic target for gastric cancer and of MET activation as a cause of resistance to c-Src inhibition. Mol Cancer Ther. 2010;9: 1188-97.

26. Correa P, Piazuelo MB, Wilson KT. Pathology of gastric intestinal metaplasia: clinical implications. Am J Gastroenterol. 2010;105:493-8.

27. Zippo A, De Robertis A, Serafini R, Oliviero S. PIM1-dependent phosphorylation of histone $\mathrm{H} 3$ at serine 10 is required for MYCdependent transcriptional activation and oncogenic transformation. Nat Cell Biol. 2007;9:932-44.

28. Macdonald A, Campbell DG, Toth R, McLauchlan H, Hastie CJ, Arthur JS. Pim kinases phosphorylate multiple sites on Bad and promote 14-3-3 binding and dissociation from Bcl-XL. BMC Cell Biol. 2006;7:1.

29. Aho TL, Sandholm J, Peltola KJ, Ito Y, Koskinen PJ. Pim-1 kinase phosphorylates RUNX family transcription factors and enhances their activity. BMC Cell Biol. 2006;7:21.

30. Wang Z, Bhattacharya N, Mixter PF, Wei W, Sedivy J, Magnuson NS. Phosphorylation of the cell cycle inhibitor p21Cip1/WAF1 by Pim-1 kinase. Biochim Biophys Acta. 2002;1593:45-55.
31. Bachmann M, Hennemann H, Xing PX, Hoffmann I, Moroy T. The oncogenic serine/threonine kinase Pim-1 phosphorylates and inhibits the activity of Cdc25C-associated kinase 1 (C-TAK1): a novel role for Pim-1 at the G2/M cell cycle checkpoint. J Biol Chem. 2004;279:48319-28.

32. Ruzinova MB, Caron T, Rodig SJ. Altered subcellular localization of c-Myc protein identifies aggressive B-cell lymphomas harboring a c-MYC translocation. Am J Surg Pathol. 2010;34:882-91.

33. Santio NM, Vahakoski RL, Rainio EM, Sandholm JA, Virtanen SS, Prudhomme M, et al. Pim-selective inhibitor DHPCC-9 reveals Pim kinases as potent stimulators of cancer cell migration and invasion. Mol Cancer. 2010;9:279.

34. Slamon DJ, Clark GM, Wong SG, Levin WJ, Ullrich A, McGuire WL. Human breast cancer: correlation of relapse and survival with amplification of the HER-2/neu oncogene. Science. 1987;235:177-82.

35. Yan B, Yau EX, Bte Omar SS, Ong CW, Pang B, Yeoh KG, et al. A study of HER2 gene amplification and protein expression in gastric cancer. J Clin Pathol. 2010;63:839-42.

36. Shay KP, Wang Z, Xing PX, McKenzie IF, Magnuson NS. Pim-1 kinase stability is regulated by heat shock proteins and the ubiquitin-proteasome pathway. Mol Cancer Res. 2005;3:170-81. 